\title{
3D Printed clamps to study the mechanical properties of tendons at low strains
}

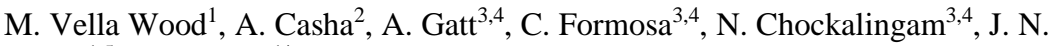
Grima $^{1,5}$ and R. Gatt ${ }^{1 *}$.

1 Metamaterials Unit, Faculty of Science, University of Malta, Msida MSD 2080, Malta

2Department of Anatomy, Faculty of Medicine \& Surgery, University of Malta, Msida MSD 2080, Malta

3Department of Podiatry, Faculty of Health Sciences, University of Malta, Msida MSD 2080, Malta 4 Faculty of Health Sciences, Staffordshire University, Science Centre, Leek Road ST4 2DF, UK

5Department of Chemistry, Faculty of Science, University of Malta, Msida MSD 2080, Malta

e-mail: ruben.gatt@um.edu.mt

\begin{abstract}
Various clamp designs have been proposed over the years with different degrees of complexity and actual performance vis-à-vis correct grip and reduced impact on the surface strains of the tendon sample. The previous studies all focused on impact on ultimate tensile strain and Young's modulus of the tendons. Here we propose an innovative clamp design that utilizes the fairly recently available technology that is $3 \mathrm{D}$ printing to produce a made-to-measure tendon sleeve that encloses the sample ends and also enables straightforward alignment within the testing equipment. All experiments were carried out using the superficial digital flexor tendons obtained from pig hind legs. The Young's moduli for the linear region of the tendon obtained using this clamp design were very similar to those obtained using the cryo Jaw by other researchers for the same tendon. Furthermore, negative Poisson's ratios were also obtained for the toe-region, in agreement with previous work.
\end{abstract}




\section{Introduction}

Understanding the mechanical characteristics that allow biological systems such as tendons to be so effective, enhances our ability to develop better man-made materials and structures, particularly materials and systems that exhibit counter intuitive properties such as auxetic behaviour ${ }^{[1-8]}$. An important material property which characterisation can lead to a better understanding of how a material behaves when uniaxially loaded is the Poisson's ratio. The Poisson's ratio can be defined mathematically as the negative of the ratio of the strain in the lateral direction to the strain in the loading direction. When studying the ex-vivo mechanical properties of biological materials such as tendons, the practically ubiquitous procedure entails clamping both ends of a tendon sample and subjecting it to controlled stretching loads in a tensile loading machine (uniaxial tensile testing). However, the external morphology of tendon being, simply put, wet soft tissue, means that there are challenges in achieving accurate tensile tests.

Sufficient compression of the tendon to hold the individual sub-fibres is necessary to reduce slippage and stress concentrations within the clamp, but in itself causes water to exude out of the tendon. The resulting reduced friction between clamp and tendon requires even more tightening of the clamps ${ }^{[9]}$. Lateral expansion of the tendon end within the clamp as well as significant damage to the structure are possible effects. On loading, the resulting mechanical properties observed might hence be dissimilar to what happens in-vivo ${ }^{[10,11]}$.

Various clamp designs, tendon treatment options and materials for wrapping the part of the tendon inside the clamp have been proposed. The current gold standard is considered to be the 'cryo-jaw' design, whereby the soft tissue ends are frozen quickly using liquid carbon dioxide or liquid nitrogen and fixed in serrated 
brass-clamps. Less compression is required which reduces the likelihood of damage to the tissue, allowing the application of high loads to the point of failure by rupture with no slippage being observed ${ }^{[9,12-15]}$, and therefore obtaining results closer to true strains than with non-frozen tendon ends ${ }^{[16]}$. However, with increasing duration of experiment, increased freezing of the tendon towards the central section was observed ${ }^{[17]}$. Other downsides include the complicated equipment requirements and the associated high cost for a cryogenic system.

Alternative clamp designs at lower cost and simpler equipment structure have been proposed. One innovative clamp design allows a tendon to be pressed firmly around the sandblasted angular clamp and preventing slippage ${ }^{[18,19]}$. Alternatively, tendons can be clamped by holding the bone at one end and clamping intramuscular tendinous fibres at the other - thereby not compressing the tendon tissue at all ${ }^{[20,21]}$. However, this is not always practical or even possible as tendons may bifurcate into sections making it impossible to obtain a single straight section attached to both bone and muscle, e.g. the porcine hind leg digital flexor tendon ${ }^{[22]}$. A more recent aluminium clamp design, whereby tendon ends are first wrapped in cloth before being clamped tightly within two serrated surfaces was reported. The flexor profundus digitorus tendons harvested from aged (70+) human cadaver hands were all reported as having ruptured towards the midsection ${ }^{[23]}$. Clamps made of plastic with symmetric interlocking, 'peak-to-valley' teeth ${ }^{[24]}$, and a titanium-alloy clamp blocks on the side to reduce lateral expansion and asymmetric, nylon, 'peak-to-peak' teeth, softer than metal, to further reduce damage to the tendon have been suggested $^{[25]}$.

Recently, it was shown that tendons may have a negative Poisson's ratio during toe-region stretching ${ }^{[26]}$. However, the clamping of these tendons may have 
resulted in flaring of the fibers within the clamp, something which may affect the measured Poisson's ratio ${ }^{[10,11]}$. In view of all this, this study aims to present a novel clamp designed to reduce or eliminate flaring of the tendon. In order to validate the suitability of this clamp, the Young's modulus of tendons (pig flexor tendons) as measured by this clamp will be compared to the Young's modulus obtained using the clamp design by Fessel ${ }^{[23]}$ as well as the various Young's modulus measurements of pig flexor tendons reported in the literature. The Poisson's ratios of the tendons as obtained from this clamp will also be reported in this study.

\section{Clamp Design}

The clamp design, which will be presented in this study, will be referred to as NFE clamp (non-flaring ends clamp). As implied by the name, the NFE clamp will be designed in such a way to reduce/remove in-clamp flaring of the tendons. This may be accomplished by having a clamp that surrounds the tendon exactly from all directions (a hand-glove approach). This ideal situation implies that for each tendon, a specific made-to-measure clamp has to be produced, something which may result difficult to achieve. This is due to the fact that producing numerous clamps using traditional manufacturing methods with materials such as aluminum or steal will result in a long production time as well as extremely high cost. In order to overcome this obstacle, we will make use of a recent technology, namely Fused Filament 3D printing. This recently developed technique has the advantages of accuracy, repeatability and speed enabling the user to print the exact sized sleeve immediately prior to use in a small amount of time and with low materials costs. In order to save both material and time, the main body of the clamp will be made out of a block of solid aluminium where a cutout is introduced (see Figure 1b). A 3D printed 'sleeve' 
(which is tailor made for a particular tendon) can be inserted in this cut-out. This will permit the re-use of the aluminum block.

The sleeve was designed in such a way as to reduce slippage by introducing perfectly interlocking serrations. This has the advantage of evenly distributing pressure on the tendon, thereby reducing the risk of stress concentrations ${ }^{[24]}$ (see Figure 1a) The lower part of the sleeve was designed so as to also allow the tendon to be centered between the interlocking serrations in order to prevent bulging of tendon fibers in the third direction after tightening, as demonstrated in Figure 1c. In summary, when designing the sleeve itself, the following were targeted:

1. A sufficient grip so as to reduce slippage.

2. Closing the sides so that the tendon ends did not flare out under tension.

3. Serration design that avoided the tendon bulging on tightening of the clamp.

4. Simple, cost-effective design.

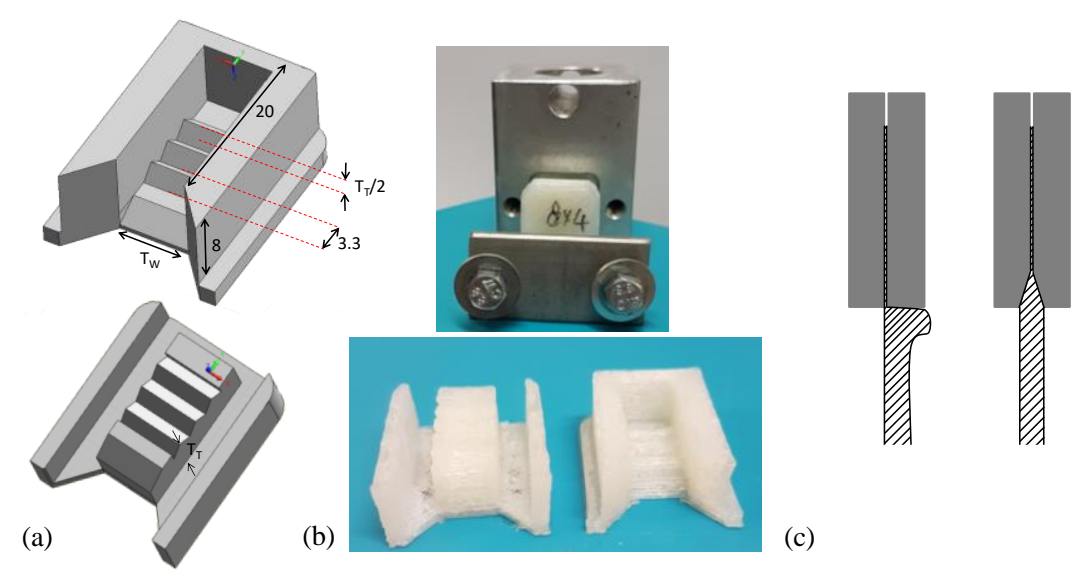

Figure 1: (a) A graphical depiction of the sleeve produced through 3D printing with the serrated ends. All measurements in the figure are in $\mathbf{m m}$ and $\mathrm{T}_{\mathrm{W}}$ stands for the width of the tendon and $T_{T}$ stands for the thickness of the tendon. (b) The clamping apparatus and an example of a 3D printed sleeve. (c) Comparative diagrams that show how bulging occurs when the tendon is squeezed between the clamp without the flared opening vs our clamp design with the flared opening. 


\section{Methodology}

The tests were all carried out on proximal sections of the superficial digital flexor tendons obtained from freshly slaughtered pig hind legs from a local abattoir. The samples were collected and tested within 12 hours of the animal death, and were stored in a refrigerator at $5^{\circ} \mathrm{C}$ in the interim period with small amount of saline used to keep the tendons from drying out. Following dissection, all connective tissue and surrounding sheaths around the tendon were removed. The tendons were then clamped in a tensile loading machine (Testometric, UK). Details of clamping procedures used for the Fessel Clamp and the NFE clamp are described below.

\subsection{Fessel clamp}

Before introducing the tendons in the Fessel clamp, the ends of the tendons were wrapped in nylon cord (see Figure 2a). The purpose of the nylon cord was to prevent the sample ends from spreading out under the pressure of the clamps and hence reduce artifacts in the recorded data. The bolts tightened to a torque of $6 \mathrm{Nm}^{-1}$.
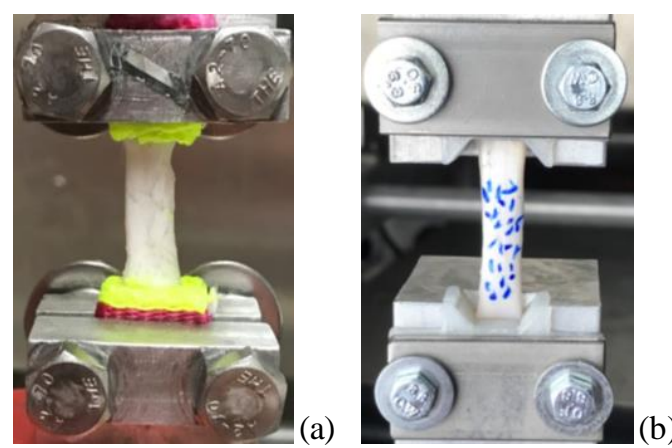

Figure 2: (a) Tendon in Fessel clamp. The nylon cord wrapping around the tendon ends can be clearly seen. (b) Tendon in NFE clamp, the 3-D printed sleeve can be clearly seen inside the aluminium block. Note also the markings on the tendon for pattern recognition. 


\subsection{NFE clamp}

The ends of the tendons were carefully measured for width and thickness using vernier calipers. The values obtained were then used to $3 \mathrm{D}$ print the exactsized sleeves using ABS plastic. Additive manufacturing was performed on a Wanhoo Duplicator 4S FFF (fused filament fabrication) 3D printer. In the case of the ABS filament, the extruder temperature was set to $230^{\circ} \mathrm{C}$ and the bed temperature was set to $105^{\circ} \mathrm{C}$. Furthermore, in order to remove the possibility of warping, Magigoo(R) 3D printing adhesive was used on the printing bed.

The tendons within the sleeves were then placed inside an aluminium block cutout. The $3 \mathrm{D}$ printed sleeve was then held in place by using a steel strip, bolted to the aluminum block (see Figure $2 \mathrm{~b}$ ), with the bolts tightened to a torque of $6 \mathrm{~N} \mathrm{~m}^{-1}$.

\subsection{Tensile loading methodology}

Tensile tests were carried out on a Testometric Universal loading machine having a $1000 \mathrm{~kg}$ F load cell (S/N 31440), and equipped with a duly calibrated camera video- extensometer (Messphysik, Germany). An initial pre-conditioning step, similar to that used by Reyes et al. ${ }^{[28]}$ and in Gatt et al. ${ }^{[26]}$, was performed where the tendon was first stretched to a $10 \mathrm{~N}$ load. This load was maintained for $30 \mathrm{~s}$ after which the load was returned to $0 \mathrm{~N}$ with a further rest period of 30 seconds. This cycle was repeated ten times. The tendon was then marked with multiple random dots using ink for use with the Messphysik pattern recognition software (see Figure $2 \mathrm{~b}$.). The actual tests were performed with a strain rate set at $5 \mathrm{~mm} / \mathrm{min}$, as was used previously by Shadwick ${ }^{[27]}$ and Gatt et al. ${ }^{[26]}$. Throughout the sample preparation and test procedure the samples were wetted with saline water at regular 
intervals to keep them moist.

For each tendon used in this study, the axial strain was determined through video extensometry, utilizing the Messphysik pattern recognition software and a tensile loading machine (Testometric, UK). Whilst using the video extensometer, the axial strains were measured from six different locations i.e. clamp to clamp (which is the same as the strain measured from the tensile loading machine), at the clamp tendon interface (for both top and bottom clamps) and at three other lengths along the tendon length as shown in Figure 3. Furthermore, transverse strains were also measured from the centre point of the tendon, at two or three different places, depending on the length of the tendon as shown in Figure $3 \mathrm{~b}$. From this data, the duration of the toe region, initial and linear Young's modulus and Poisson's ratio for the plane of the tendon parallel to the bone, were then determined as explained in the supplementary information.

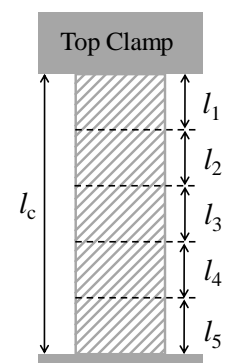

(a) Bottom Clamp

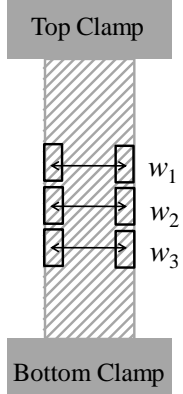

(b) Bottom Clamp

Figure 3: (a) The different locations where the axial strains were measured (b) The approximate locations where the transverse strains were measured.

\subsection{Statistics}

Using Microsoft Excel, Two-Sample t-Test Assuming Unequal Variances was performed with the Null hypothesis being that the averages of the moduli 
obtained for each clamp type are the same.

\section{$4 \quad$ Results and Discussion}

A visual analysis of the clamped tendons indicates that the tendon ends flare and bulge appreciably when the Fessel clamp is used, as shown Figure 4 (a).

However, there is no evidence of flaring or bulging of the tendon when it is fixed to the universal lading machine using the NFE clamp, as shown in Figure 4 (b).

Furthermore, in the case of the NFE clamp, referring to Figure 4 (e), one may also observe that after removing the tendon from the clamp, there is no appreciable flaring of the tendon.

As detailed in the Methodology section, the axial strain was either measured along the whole tendon sample (from clamp to clamp) or it was taken from regions within the tendon. Referring to Figure 5, which show the stress strain curves for various tendon samples, as obtained using the ABS NFE clamp and Fessel clamp, one may observe that the overall profile on the plot depends on the location from which the axial strain was measured. In fact, when taking into consideration the average strain along the whole tendon sample, the tendon seems to undergo high deformations (in the region of $15 \%$ axial strain). However, this is not the case when calculating the axial strain from the middle of the tendon sample. In this case, the axial strains are much smaller, in the region of $3 \%$. 
(a)

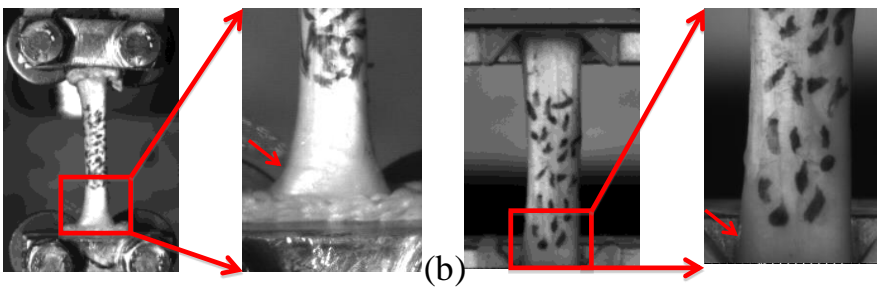

(c)

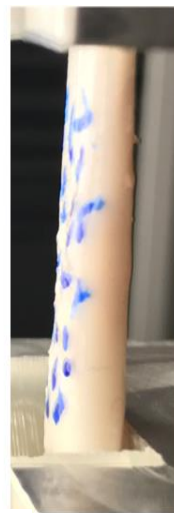

(d)

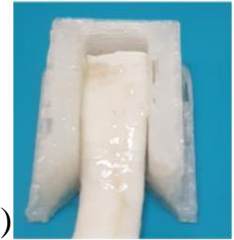

(e)
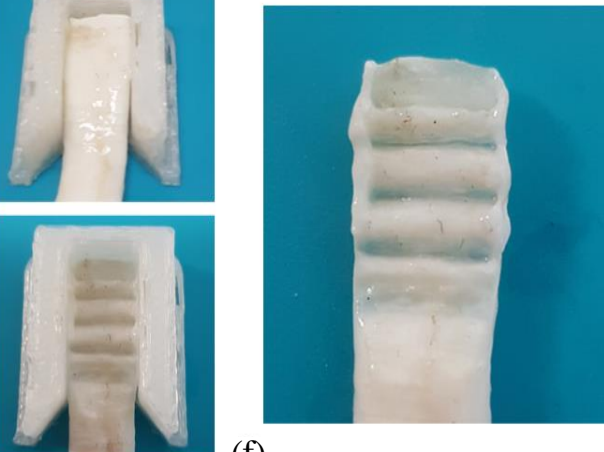

(f)

Figure 4: (a) Photo of tendon in the Fessel clamp, exhibiting flaring and bulging. (b) Photo of tendon in the NFE clamp, with no flaring or bulging observed. (c) Photo of the clamped tendon in the third direction showing that for the sleeves produced, there was no bulging of the tendon in the third direction (d) the tendon inside the sleeve before tightening the clamp (e) the tendon inside the sleeve after tightening the clamp (f) tendon removed from the clamp after tightening, showing that no flaring occurred.

Referring to Figure 6, one may note that this distinction may be due to the fact that the tendon tends to deform to a high degree at the upper and lower clamping sites when compared to the rest of the tendon. It is interesting to note that in the case of the ABS NFE clamp and the Fessel clamp, regions 11 and 15 (i.e. the regions which were closest to the upper and lower clamp respectively) deformed to a higher degree than the rest of the tendon. As discussed by Shadwick ${ }^{[27]}$, this means that taking the strain from the centre of the tendon probably gives a better representation of the tendon's stress strain relationship. 


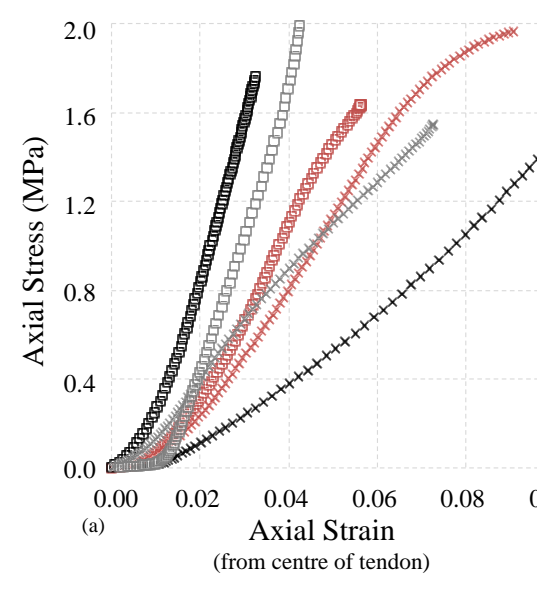

2.0

$\square \mathrm{S} 1 \times \mathrm{S} 2 \times \mathrm{S} 3 \quad \square \mathrm{S} 4 \times \mathrm{S} 5 \quad \square \mathrm{S} 6$

2.0

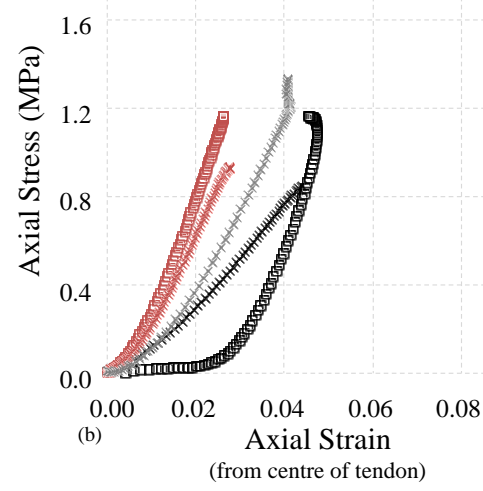

2.0

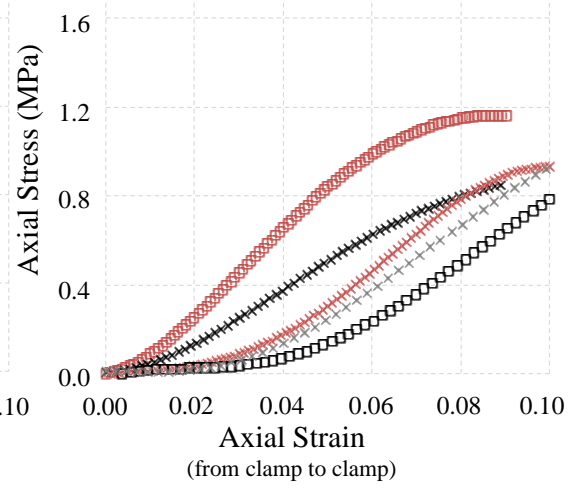

$\square \mathrm{S} 1 \times \mathrm{S} 2 \times \mathrm{S} 3 \quad \square \mathrm{S} 4 \times \mathrm{S} 5$

Figure 5: Stress strain curves for the tendon samples used in this study where the axial data was taken either from the centre of the tendon or from clamp to clamp, as duly noted on the figure. (a) data obtained using the ABS NFE clamp and (b) data obtained using the Fessel clamp.

Further analysis of the stress strain plots (Figure 5) indicates that there is some variability in the duration of the toe region. Referring to Figure 6(d), there seems to be no relation between the sample length and the duration of the toe region, with the duration of the toe region being between $1.2 \%$ and $3.2 \%$ for most of the samples tested. The calculated transition point strains from toe-region to linear region are presented Figure 6(c). This is comparable to the values found in literature, with 
Shadwick and co-workers reporting the toe to linear region transition at approximately $4 \%$ (0.04 strain) for porcine deep digital flexor tendons ${ }^{[27]}$, while Reyes et al. reported this transition at about 4 to $8 \%$ (grip-to-grip) strain -for superficial digital flexor tendons ${ }^{[28]}$.
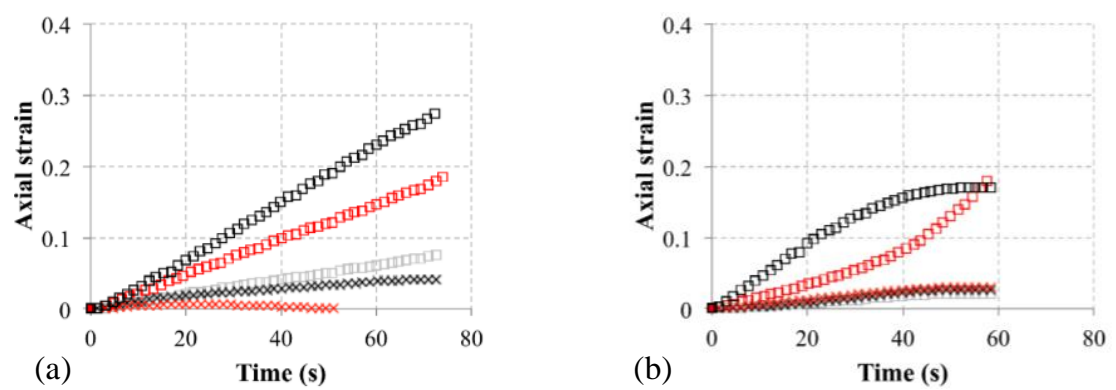

$\square l_{1} \quad \square l_{2} \times l_{3} \times l_{4} \quad \square l_{5}$

\begin{tabular}{|c|c|c|c|}
\hline \multicolumn{2}{|c|}{ ABS NFE Clamp } & \multicolumn{2}{|c|}{ Fessel Clamp } \\
\hline S1 & 0.023 & S1 & 0.012 \\
\hline S2 & 0.028 & $\mathrm{~S} 2$ & 0.021 \\
\hline S3 & 0.028 & S3 & 0.010 \\
\hline S4 & 0.017 & S4 & 0.032 \\
\hline S5 & 0.012 & S5 & 0.021 \\
\hline S6 & 0.018 & & \\
\hline
\end{tabular}

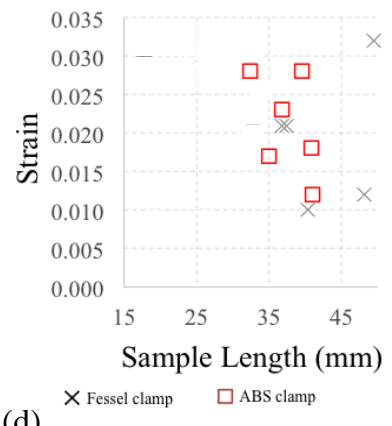

Figure 6: Axial strain against time plots as measured from different regions of the tendon (a) ABS NFE Clamp and (b) Fessel Clamp. The equivalent plots for the other samples may be found in the supplementary information. (c) The strain at the Toe-region to Linear region Transition point for the tendon samples. (d) Plot showing the duration of the toe region versus the sample length.

The toe region and linear region Young's Moduli obtained are tabulated in Table 1.

The linear region Young's moduli measured in this study may be evaluated within the range of results presented in the literature. Reyes and co-workers measured the Young's modulus of hind leg superficial and deep pig digital flexor tendon explants and obtained values of $0.29(0.15) \mathrm{GPa}$ and $0.25(0.08) \mathrm{GPa}$ respectively ${ }^{[28]}$. Other 
reported values include the work carried out by Shadwick who used a Cryo Jaw for the front, deep digital flexor tendon of pigs to obtain a value of $1.66(0.16) \mathrm{GPa}^{[27]}$, and similarly by De Zee et al who reported 0.85 (0.20) GPa for the same tendon and experiment set up, excluding the tendon end freezing ${ }^{[29]}$. Additionally, values of 0.9 GPa (unspecified pig leg tendons) ${ }^{[30]}$ and approximately $0.3-0.8 \mathrm{GPa}$ (values read off a graph for unspecified pig leg tendons) ${ }^{[31]}$ were also reported. This means that the results obtained through the clamp designed in this work are consistently within the same order of magnitude with previously obtained results including those obtained using a Cryo Jaw albeit using a simpler and likely lower cost set up. Here one must take into account the caveat that individual tendons biomechanical properties are affected by 'stress-in-life' and therefore a fairly high degree of variability is to be expected ${ }^{[32]}$.

\section{Table 1: Average Young's Moduli}

\begin{tabular}{|l|l|l|l|l|}
\hline \multicolumn{3}{|l|}{ ABS NFE Clamp* } & \multicolumn{2}{l|}{ Fessel Clamp* } \\
\hline $\begin{array}{l}\text { Toe } \\
\text { Region }\end{array}$ & $\begin{array}{l}\text { Young's } \\
\text { Modulus/ GPa }\end{array}$ & $\mathrm{R}^{2}$ & $\begin{array}{l}\text { Young's } \\
\text { Modulus/GPa }\end{array}$ & $\mathrm{R}^{2}$ \\
\hline $\begin{array}{l}\text { Average } \\
\text { Standard } \\
\text { deviation }\end{array}$ & 0.11 & 0.96 & 0.12 & 0.99 \\
\hline $\begin{array}{l}\text { Linear } \\
\text { Region }\end{array}$ & $\begin{array}{l}\text { Young's } \\
\text { Modulus/ GPa }\end{array}$ & $\mathrm{R} 2$ & $\begin{array}{l}\text { Young's } \\
\text { Modulus/ GPa }\end{array}$ & $\mathrm{R} 2$ \\
\hline $\begin{array}{l}\text { Average } \\
\text { Standard } \\
\text { deviation }\end{array}$ & 0.43 & 1.00 & 0.42 & 1.00 \\
\hline
\end{tabular}

From Table 1, one may infer that the two clamps produced similar results for toeregion Young's modulus, albeit the high variability in the results obtained. The results obtained from a Two-Sample t-Test Assuming Unequal Variances test (see supplementary information) confirm that up to a level of $95 \%$ confidence, the Young's moduli obtained for the toe- and linear regions of the stress strain curve are the same regardless of the clamp used. Here it must be stressed out that this is an 
initial evaluation of the designed clamp and therefore further testing needs to be carried out.

An analysis of the strain-strain plots obtained using the new clamp design (See

Figure 7) showed a consistent positive gradient within the first $2 \%$ strain (indicating a negative Poisson's ratio), in accordance with Gatt et al. ${ }^{[26]}$ who used the clamp designed by Fessel and co-workers ${ }^{[23]}$ to measure the Poisson's ratio. Furthermore, the results obtained in this study also suggest that as the axial strain increases, the gradient of the strain-strain plot gradually became less positive, indicating that the Poisson's ratio is becoming less negative. This is also in agreement with the mechanism proposed by Gatt et al., wherein, negative Poisson's ratio was explained in terms of the 'opening up' of the crimp structure of the tendon (similar to the way that negative Poisson's ratio is obtained when stretching a piece of crumpled paper) [26, 32]. The fact that the new clamp was designed to prevent the tendon ends from spreading, further suggests that the observed negative Poisson's ratio in the Coronal plane of the superficial digital flexor tendon is an actual property of the tendon rather than a measurement artifact.
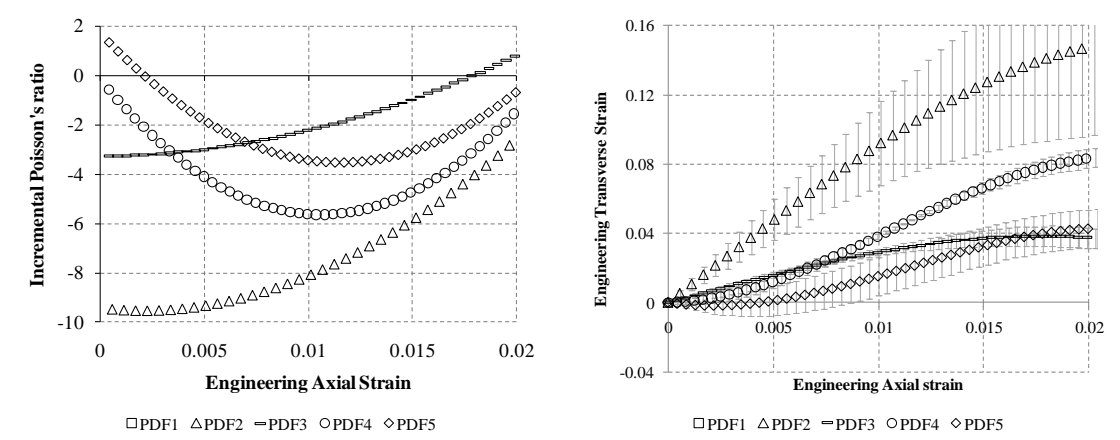


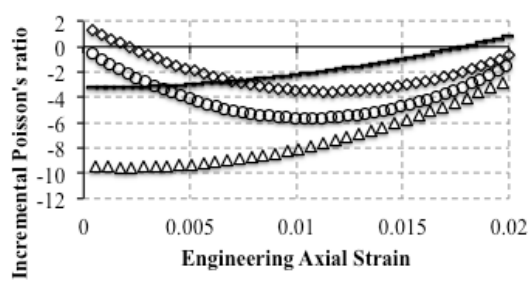

DPDF1 $\triangle$ PDF2 - PDF3 OPDF4 PPDF5

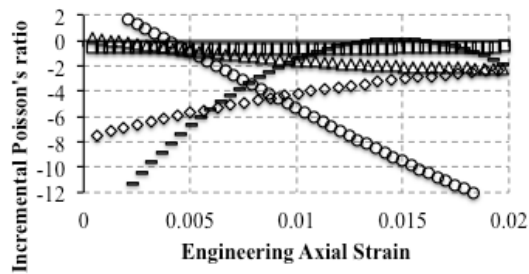

口PDF6 $\triangle$ PDF7 $=$-PDF8 OPDF9 $\triangle$ PDF10

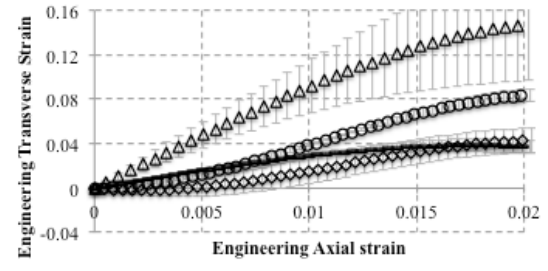

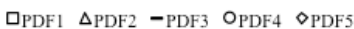

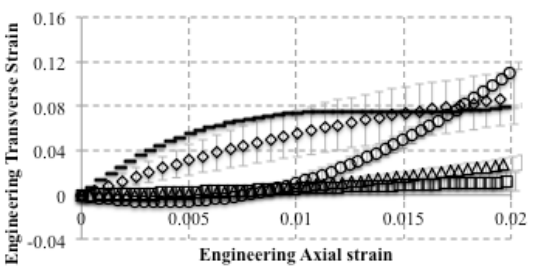

口PDF6 $\triangle$ PDF7 -PDF8 OPDF9 $\diamond$ PDF10

Figure 7: Plots showing the incremental Poisson's ratio versus the engineering axial strain (taken from the centre of the tendon) and the transverse strain against the axial strain plot for the same samples (PDF1-5 are the samples tested using the ABS NFE clamp while PDF6-10 are the samples tested using the Fessel clamp).

Before concluding, it is important to note that the 3D printed clamp proposed here has other advantages. In particular, when using this clamp design, alignment of the tendon was very easy since the dedicated sleeves ensured exact linear alignment of the tendon within the clamp. Furthermore, in the case of this setup, there is no need to wrap ${ }^{[23,33]}$ or freeze ${ }^{[9]}$ the tendon ends thereby greatly simplifying the procedure.

\section{Conclusion}

In this study, a novel but simple clamp for tensile testing of soft biological materials such as tendons was proposed and tested. It was shown that this clamp eliminates tendon-end fraying as the clamp is tightened, something, which may be result 
important when measuring the Poisson's ratio of tendons. It was shown the linear region Young's moduli for tendons obtained using the proposed clamp, is in agreement with measurements obtained when using the clamp designed by Fessel as well as those reported in literature when using the 'gold standard' clamp design i.e. the Cryo-jaw. The Poisson's ratio trends obtained for the toe-region were again negative, thus lending further credence to the tendon behaviour observed and documented in previous work.

\section{Acknowledgements:}

The authors would like to express their gratitude to the Ministry for sustainable development, the Environment and Climate Change - Public Abattoir Operations and Mr. Emanuel Schembri and Dr. Gianluca Esposito for all their help in obtaining the porcine samples.

\section{References}

1. K. W. Wojciechowski, Mol. Phys. 1987, 61, 1247

2. R. S. Lakes, Science 1987, 235, 1038.

3. K. W. Wojciechowski, Phys. Lett. A 1989, 137, 60.

4. K. E. Evans, M. A. Nkansah, I. J. Hutchinson, S. C. Rogers, Nature 1991, $253,124$.

5. K. L. Alderson, A. Alderson, K. E. Evans, J. Strain Anal. Eng. Design. 1997, 32, 201.

6. J. N. Grima, R. Jackson, A. Anderson, K. E. Evans, Adv. Mater. 2000, 12, 1912. 
7. S. Jacobs, C. Coconnier, D. DiMaio, F. Scarpa, M. Toso, J. Martinez, Smart Mater. Struct. 2012, 21, 075013.

8. R. Gatt, L. Mizzi, J. I. Azzopardi, K. M. Azzopardi, D. Attard, A. Casha,J. Briffa, J. N. Grima, Sci. Rep. 2015, 5, 8395.

9. D.J. Riemersa, H.C. Schamhardt, J Biomech. 1982, 15(8), 619.

10. R.F. Ker, in Biomechanics-Materials: A Practical Approach (Ed: J.F.V.Vincent), Oxford University Press, New York 1992, pp. 75-98.

11. R.E. Shadwick, in Biomechanics-Materials: A Practical Approach (Ed: J.F.V.Vincent), Oxford University Press, New York 1992, pp. 133-164.

12. M. Rickert, H.Georgousis, U. Witzel, Unfallchirurg. 1998, 101(4), 265.

13. M. Rickert, U. Witzel, R. Kölbel, H. Georgousis, Unfallchirurg, 2002, 105, 23.

14. F. Morelli, A. Ferretti, F. Conteduca, F. Nanni, L. Monteleone, M. Valente, $J$ Appl. Biomech. 2002, 18(4), 384.

15. P. Wieloch, G. Buchmann, W. Roth, M. Rickert, J Biomech. 2004, 37(11), 1719.

16. G.L. Matthews, K.G. Keegan, H.L. Graham, Am J Vet Res. 1996, 57(1), 111.

17. H.U. Stäubli, L. Schatzmann, P. Brunner, L. Rincón, L.P. Nolte, Am J Sports Med. 1999, 27(1), 27.

18. S. L.-Y. Woo, M.A. Gomez, Y. Seguchi, C.M. Endo, W.H. Akeson, J Orthop. Res. 1983, 1, 22.

19. K.H. Svendsen, G. Thomson, J Biomech. 1984,17(3), 225

20. G.A.C. Murrel, E.G. Lilly, R.D. Goldner, A.V. Seaber, T.M. Best. J Orthop. Res. 1994, 12, 582. 
21. A. Probst, D. Palmes, H. Freise, M. Langer, A. Joist, H.U. Spiegel, J Invest. Surg. 2000, 13(6), 313.

22. V.L.C. Feitosa, M.A.M. Esquisatto, P.P. Joazeiro, L. Gomes, S.L. Felisbino E.R. Pimentel, Braz. J Morphol. 2005, 22, 113

23. G. Fessel, K. Frey, A. Schweizer, M. Calcagni, O. Ullrich, J.G. Snedeker, Ann. Anat. 2011, 193, 237.

24. J. T.-M. Cheung, M. Zhang, Med. Eng. Phys. 2006, 28(4), 379

25. D. Shi, D. Wang, C. Wang, A.A. Liu, Med. Eng. Phys. 2012, 34(4), 516

26. R. Gatt, M. Vella Wood, A. Gatt, F. Zarb, C. Formosa, K.M. Azzopardi, A. Casha, T.P. Agius, P. Schembri-Wismayer, L. Attard, N. Chockalingam, J.N. Grima, Acta Biomater. 2015, 24, 201

27. R.E. Shadwick, J Appl Physiol. 1990, 68(3), 1033.

28. A.M. Reyes, H. Jahr, H.T.M. van Schie, H. Weinans, A.A. Zadpoor, J Mech. Behav. Biomed. Mater. 2014, 30, 324.

29. M. De Zee, F. Bojsen-Møller, M. Voigt, J Appl. Phys. 2000, 89(4), 1352

30. S.L-Y. Woo, M.A. Ritter, D. Amiel, T. Sanders, M.A. Gomez, S.C. Kuei, S. R. Garfin, W.H. Akeson, Connect. Tissue Res. 1980, 7, 177

31. M.B. Bennett, R.F. Ker, N.J. Dimery, N. Alexander, J Zool. London (A), 1986, 209, 537

32. J.N. Grima, S. Winczewski, L. Mizzi, M.C. Grech, R. Cauchi, R. Gatt, D. Attard, K.W. Wojciechowski, J. Rybicki, Adv. Mater. 2015, 27, 1455

33. B.H. Ng, S.M. Chou, B.H. Lim, A. Chong, Proc. Inst. Mech. Eng. 2005, 219, 387 